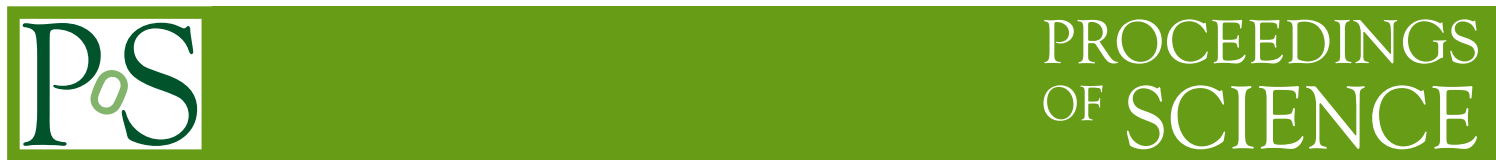

\title{
Photopion physics at MAMI
}

\author{
David Hornidge* \\ Mount Allison University \\ E-mail: dhornidge@mta.ca
}

Recent measurements and future plans for photopion experiments with the CB-TAPS detector system in the A2 hall at the Mainzer Microtron were presented. First, a measurement with linearly polarized photons and an unpolarized liquid-hydrogen target was discussed. The beam asymmetry along with differential cross sections provide the most stringent test to date of the predictions of Chiral Perturbation Theory and its energy region of convergence [1]. Second, a more recent measurement was performed using both circularly polarized photons and a transversely polarized butanol frozen-spin target to extract the polarization-dependent differential cross section associated with the target asymmetry [2]. Results from both measurements have been used for a model independent determination of $S$ - and $P$-wave multipoles in the $\pi^{0}$ threshold region, which includes for the first time a direct determination of the imaginary part of the $E_{0^{+}}^{\pi^{0} p}$ multipole. Finally, plans for a novel $\pi^{0}$ photoproduction measurement on ${ }^{3} \mathrm{He}$ to obtain the elusive $E_{0^{+}}^{\pi^{0} n}$ multipole for the neutron channel will be introduced.

The 8th International Workshop on Chiral Dynamics

29 June 2015 - 03 July 2015

Pisa, Italy

${ }^{*}$ Speaker. 


\section{Introduction}

Photoproduction of neutral pions on the proton is an important test of Chiral Perturbation Theory (ChPT), an effective field theory with the pion and nucleon as effective degrees of freedom. Because ChPT is based on the symmetries of QCD, it amounts to testing the strong interaction at low energies, i.e. in the non-perturbative, or confinement, regime. In its domain of validity, $\mathrm{ChPT}$ represents predictions of QCD subject to the errors imposed by uncertainties in the low-energy constants and by neglect of higher order terms, and so any discrepancy that is significantly larger than the combined experimental and theoretical errors must be taken seriously.

One technique for comparing experimental results to ChPT and other theoretical approaches is through a model-independent partial-wave analysis by extracting multipoles. Our efforts have been to focus on accurate measurements of low-energy $\gamma N \rightarrow \pi^{0} N$ reactions, including the sensitive spin observables that allow a unique separation of the $S$ - and $P$-waves, to perform test of these predictions. More specifically, we have conducted two separate experiments on the proton using 1) a linearly polarized photon beam with unpolarized liquid-hydrogen target [1], and 2) circularly polarized beam and a transversely polarized frozen-spin butanol target [2]. Finally, we report on plans to use an active, high-pressure gaseous ${ }^{3} \mathrm{He}$ target to access the $S$-wave amplitude on the neutron.

\section{Partial-Wave Analysis}

For any two-body photoproduction experiment with either polarized and unpolarized beam and polarized or unpolarized target, there are 16 possible observables, listed in Table 1 . However,

Table 1: Spin observables in two-body photoproduction.

\begin{tabular}{c|cccc} 
set & \multicolumn{4}{|c}{ observables } \\
\hline single & $d \sigma / d \Omega$ & $\Sigma$ & $\mathrm{T}$ & $\mathrm{P}$ \\
beam-target & $\mathrm{G}$ & $\mathrm{H}$ & $\mathrm{E}$ & $\mathrm{F}$ \\
beam-recoil & $\mathrm{Ox} \mathrm{yz}^{\prime}$ & $\mathrm{Cx}$ & $\mathrm{Cz}$ \\
target-recoil & $\mathrm{Tx}$ & $\mathrm{Tz}$ & $\mathrm{Lx} \mathrm{x}^{\prime}$ & $\mathrm{Lz}$
\end{tabular}

a carefully chosen set of eight independent observables is enough for a complete description of an experiment, but for a complete partial-wave analysis, one needs fewer observables, and with only four one can obtain solutions with only discrete sign ambiguities. Moreover, below the $2 \pi$ threshold, we only need two observables, the differential cross section $d \sigma / d \Omega$ and beam asymmetry $\Sigma$, and unitarity. If we make use of a third single-polarization observable, the target asymmetry $T$, we do not need unitarity. We have used both empirical single-energy and energy-dependent fits to $d \sigma / d \Omega, \Sigma$, and $T$, extracted coefficients and multipoles, and then compared them to theory. Again, see Refs. [1,2] and references contained therein for further details.

In the threshold region, there is one $S$-wave $(l=0)$ multipole, three $P$-wave $(l=1)$ multipoles, and four $D$-wave $(l=2)$ multipoles that contribute: 


$$
\begin{array}{ll}
l=0 & E_{0^{+}}^{\pi^{0} p} \\
l=1 & E_{1^{+}}, M_{1^{+}}, M_{1^{-}} \\
l=2 & E_{2^{+}}, E_{2^{-}}, M_{2^{+}}, M_{2^{-}}
\end{array}
$$

and in alternate notation we have

$$
\begin{aligned}
P_{1} & =3 E_{1^{+}}+M_{1^{+}}-M_{1^{-}} \\
P_{2} & =3 E_{1^{+}}-M_{1^{+}}+M_{1^{-}} \\
P_{3} & =2 M_{1^{+}}+M_{1^{-}} \\
P_{23}^{2} & =\frac{1}{2}\left(P_{2}^{2}+P_{3}^{2}\right)
\end{aligned}
$$

and

$$
\begin{aligned}
& D_{1}=E_{2^{-}}-3 M_{2^{-}}+6 E_{2^{+}}+3 M_{2^{+}} \\
& D_{2}=E_{2^{-}}-M_{2^{-}}-4 E_{2^{+}}+M_{2^{+}} \\
& D_{3}=2 M_{2^{-}}+3 M_{2^{+}} \\
& D_{4}=E_{2^{-}}+M_{2^{-}}+E_{2^{-}}-M_{2^{+}} .
\end{aligned}
$$

In the threshold region, the $P$ - and $D$-wave amplitudes are assumed to be purely real, whereas due to rescattering that occurs above $\pi^{+}$threshold, as show in Figure 1, the $S$-wave amplitude has an

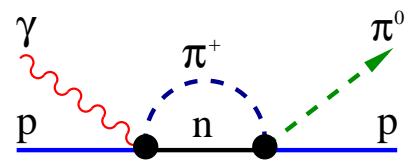

Figure 1: Rescattering above $\pi^{+}$threshold in the $\gamma p \rightarrow \pi^{0} p$ reaction.

imaginary component and there exists a so called unitarity cusp

$$
\begin{aligned}
E_{0^{+}}^{\pi^{0} p} & =\operatorname{Re} E_{0^{+}}^{\pi^{0} p}+i \operatorname{Im} E_{0^{+}}^{\pi^{0} p} \\
& =\operatorname{Re} E_{0^{+}}^{\pi^{0} p}+i \beta \frac{q_{\pi^{+}}}{m_{\pi^{+}}}
\end{aligned}
$$

where $\beta$ is the cusp function and $q_{\pi^{+}}$is the c.m. momentum of the rescattered $\pi^{+}$. Note that according to unitarity, the cusp function can be written in terms of the $S$-wave amplitude for the $\gamma p \rightarrow \pi^{+} n$ reaction, $E_{0^{+}}^{\pi^{+} n}$, and the scattering length $a_{e x}\left(\pi^{+} n \rightarrow \pi^{0} p\right)$ :

$$
\beta=E_{0^{+}}^{\pi^{+}} a_{e x}\left(\pi^{+} n \rightarrow \pi^{0} p\right) \text {. }
$$

In principle, if one could make an accurate enough determination of $\operatorname{Im} E_{0^{+}}^{\pi^{0} p}$, then using unitarity and the known value of $E_{0^{+}}^{\pi^{+} n}$ one could test strong-isospin breaking, since

$$
a_{e x}\left(\pi^{+} n \rightarrow \pi^{0} p\right)=a_{e x}\left(\pi^{-} p \rightarrow \pi^{0} n\right) .
$$

It is only about a $2 \%$ effect, though, so precise data with low systematic errors are necessary. 


\subsection{Single-Energy Fits}

Including only $S$ - and $P$-waves, empirical single-energy fits to the multipoles can be made to the differential cross section and beam asymmetry using the following expressions:

$$
\begin{gathered}
\frac{d \sigma}{d \Omega}(\theta)=\frac{q}{k}\left(a_{0}+a_{1} \cos \theta+a_{2} \cos ^{2} \theta\right) \\
\frac{d \sigma}{d \Omega}(\theta) \Sigma(\theta)=\frac{q}{k} \sin ^{2} \theta b_{0}
\end{gathered}
$$

where $k(q)$ is the c.m. photon (pion) momentum, $\theta$ is the c.m. pion scattering angle, and the coefficients are given by

$$
\begin{aligned}
& a_{0}=\left|E_{0^{+}}^{\pi^{0} p}\right|^{2}+P_{23}^{2} \\
& a_{1}=2 \operatorname{Re} E_{0^{+}}^{\pi^{0} p} P_{1} \\
& a_{2}=P_{1}^{2}-P_{23}^{2} \\
& b_{0}=\frac{1}{2}\left(P_{3}^{2}-P_{2}^{2}\right) .
\end{aligned}
$$

In this way we have four measured quantities, $a_{0}, a_{1}, a_{2}, b_{0}$, and four unknown real parameters: $\operatorname{Re} E_{0^{+}}^{\pi^{0} p}, P_{1}, P_{2}, P_{3}$, assuming that $\operatorname{Im} E_{0^{+}}^{\pi^{0} p}$ is taken from unitarity. Note that $D$-waves contribute, but they are small and can be added using the Born terms. Including them, we have

$$
\begin{gathered}
\frac{d \sigma}{d \Omega}(\theta)=\frac{q}{k}\left(a_{0}+a_{1} \cos \theta+a_{2} \cos ^{2} \theta+a_{3} \cos ^{3} \theta+a_{4} \cos ^{4} \theta\right) \\
\frac{d \sigma}{d \Omega}(\theta) \Sigma(\theta)=\frac{q}{k} \sin ^{2} \theta\left(b_{0}+b_{1} \cos \theta+b_{2} \cos ^{2} \theta\right)
\end{gathered}
$$

where we now have eight coefficients

$$
\begin{aligned}
& a_{0}=\left|E_{0^{+}}^{\pi^{0} p}\right|^{2}+P_{23}^{2}+\operatorname{Re} E_{0^{+}}^{\pi^{0} p} D_{1}+\frac{1}{4}\left(D_{1}^{2}+9 D_{2}^{2}\right) \\
& a_{1}=2 \operatorname{Re} E_{0^{+}}^{\pi^{0} p} P_{1}-P_{1} D_{1}-3 P_{2} D_{2}+3 P_{3} D_{3} \\
& a_{2}=P_{1}^{2}-P_{23}^{2}-\frac{3}{2}\left(D_{1}^{2}-3 D_{2}^{2}-3 D_{3}^{2}+3 D_{4}^{2}\right)+3 \operatorname{Re} E_{0^{+}}^{\pi^{0} p} D_{1} \\
& a_{3}=3\left(P_{1} D_{1}+P_{2} D_{2}-P_{3} D_{3}\right) \\
& a_{4}=\frac{9}{4}\left(D_{1}^{2}-2 D_{2}^{2}-2 D_{3}^{2}+D_{4}^{2}\right) \\
& b_{0}=\frac{1}{2}\left(P_{3}^{2}-P_{2}^{2}-3 D_{1} D_{4}\right)+3 \operatorname{Re} E_{0^{+}}^{\pi^{0} p} D_{4} \\
& b_{1}=3\left(P_{1} D_{4}+P_{2} D_{2}+P_{3} D_{3}\right) \\
& b_{2}=\frac{9}{2}\left(-D_{2}^{2}+D_{3}^{2}+D_{1} D_{4}\right) .
\end{aligned}
$$

If one can measure the transverse target asymmetry, $T$, the imaginary part of the $S$-wave amplitude can be accessed:

$$
T=\operatorname{Im} E_{0^{+}}^{\pi^{0} p}\left(P_{3}-P_{2}\right) \sin \theta .
$$

In addition to our measurement of the differential cross sections and beam asymmetry, here we report on the very first measurement of $T$ in neutral pion photoproduction near threshold. 


\subsection{Empirical Energy-Dependent Fits}

Instead of extracting the multipoles at each incident photon energy, one can expanded them as a function of $W$ by fitting the coefficients using the following ansatz for the $S$-wave:

$$
E_{0^{+}}(W)=E_{0^{+}}^{(0)}+E_{0^{+}}^{(1)}\left[\frac{k_{\gamma}(W)-k_{\gamma, \mathrm{thr}}}{m_{\pi^{+}}}\right]+i \beta \frac{q_{\pi^{+}}(W)}{m_{\pi^{+}}}
$$

and the $P$-waves:

$$
P_{i}(W)=\frac{q_{\pi^{0}}(W)}{m_{\pi^{+}}}\left\{P_{i}^{(0)}+P_{i}^{(1)}\left[\frac{k_{\gamma}(W)-k_{\gamma, \mathrm{thr}}}{m_{\pi^{+}}}\right]\right\} .
$$

Here $k_{\gamma}$ and $k_{\gamma, \text { thr }}$ are in the lab frame, and $q_{\pi^{0}}\left(q_{\pi^{+}}\right)$is the c.m. $\pi^{0}\left(\pi^{+}\right)$momentum. Note that the superscripts (0),(1) denote intercept and slope, respectively. Again, the $D$-waves are added by hand using the Born terms.

\section{Proton}

\subsection{Beam Asymmetry}

We have measured the linear beam asymmetry, $\Sigma$, and differential cross section, $d \sigma / d \Omega$, in neutral pion photoproduction from the proton near threshold. More details can be found in Ref. [1]. The data were taken in the A2 hall at the Institute for Nuclear Physics in Mainz, Germany, using the CB-TAPS detector system. The specific run parameters are given in Table 2. Note that a

Table 2: Run parameters for the beam-asymmetry measurement.

\begin{tabular}{l|c} 
PARAMETER & VALUE \\
\hline Electron Beam Energy & $855 \mathrm{MeV}$ \\
Target & $10-\mathrm{cm} \mathrm{LH}_{2}$ \\
Radiator & $100 \mu \mathrm{m} \mathrm{Diamond}$ \\
Tagged Energy Range & $100-800 \mathrm{MeV}$ \\
Channel Energy Resolution & $2.4 \mathrm{MeV}$ \\
Polarization Edge & $\sim 190 \mathrm{MeV}$ \\
Degree of Polarization & $40-70 \%$ \\
Beam on Target & $90 \mathrm{~h} \mathrm{Full}+20 \mathrm{~h}$ Empty
\end{tabular}

previous measurement was conducted with the TAPS detector alone [3], but due to the poor solidangle coverage and a problem with unsubtracted empty-target contributions, the results had larger systematic errors than originally reported.

A plan view of the Mainzer Microtron (MAMI) is shown in Figure 2 and one can readily see the three race-track microtrons (RTMs) and the harmonic double-sided microtron (HDSM), although the latter was not used in this experiment. The linearly polarized photon beam was produced using the Glasgow-Mainz tagger and a diamond radiator. The CB-TAPS detector system, with a GEANT4 view shown in Figure 4, consists of the Crystal Ball (CB) detector and the TAPS detector. The CB consists of NaI scintillators arranged around the target, covering $93 \%$ of $4 \pi$ steradians, 
Figure 2: Plan view of the MAMI accelerator.

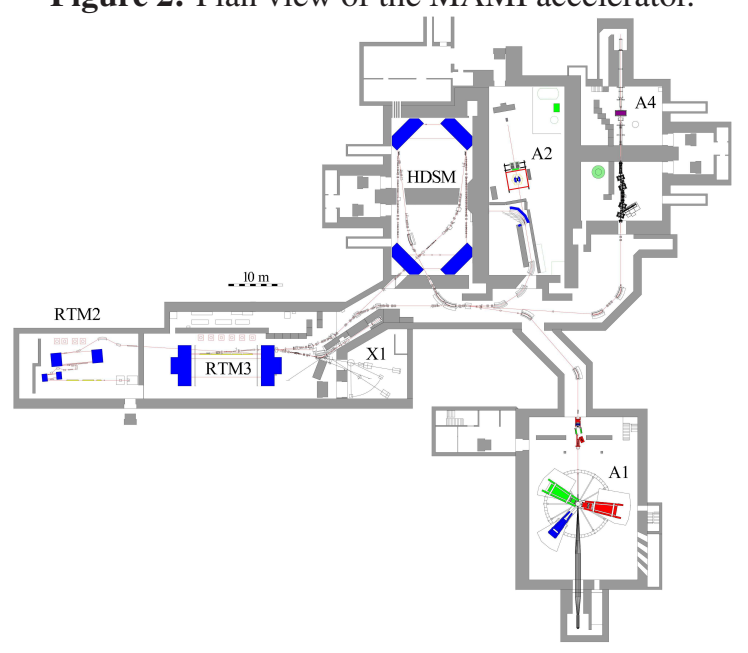

Figure 3: The Glasgow-Mainz photon tagging spectrometer.

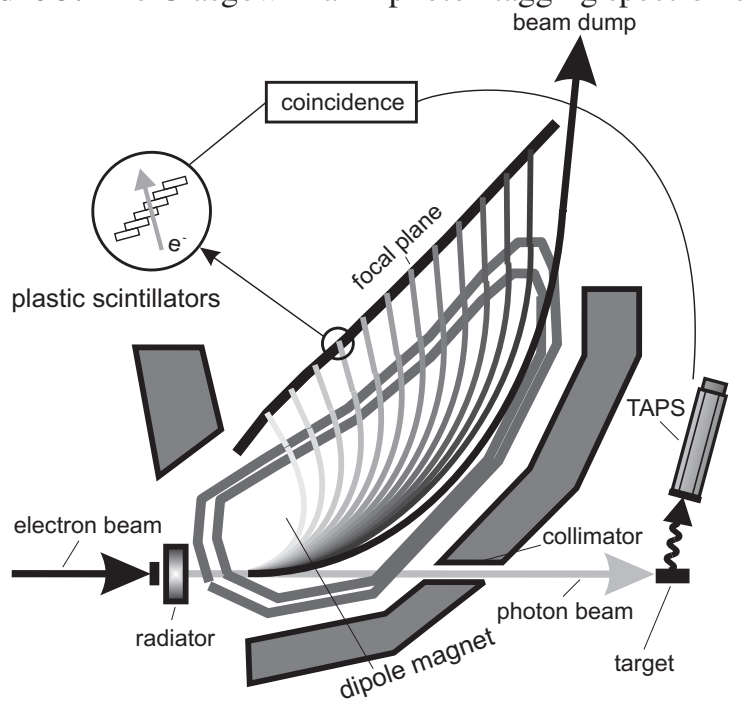

and at the centre between the target cell and $\mathrm{CB}$ was the veto barrel particle-identification detector (PID). TAPS, composed of both $\mathrm{BaF}_{2}$ and $\mathrm{PbWO}_{4}$ modules, each with an individual veto, was positioned downstream of the target to cover the all-important forward region. Together the $\mathrm{CB}$ and TAPS cover $96 \%$ of $4 \pi$ steradians. The unpolarized liquid-hydrogen $\left(\mathrm{LH}_{2}\right)$ target was relatively complicate due to the snout configuration necessary to fit inside the PID and CB.

Results of our measurement are shown in Figures 5, and 6. Note the excellent statistics in both $d \sigma / d \Omega$ and $\Sigma$, and for the first time, energy dependence of $\Sigma$. There is good agreement with both HBChPT (black) [4] and relativistic ChPT (blue) [5], and the empirical fit is also shown with statistical error band (green). For the very first time we have obtained the energy dependence of the $P$-waves, as well as the energy region of agreement for Chiral Perturbation theory, both HBChPT and relativistic ChPT. 
Figure 4: Cut-away GEANT4 view of the CB-TAPS detector system showing the Crystal Ball, TAPS, and the PID detectors.

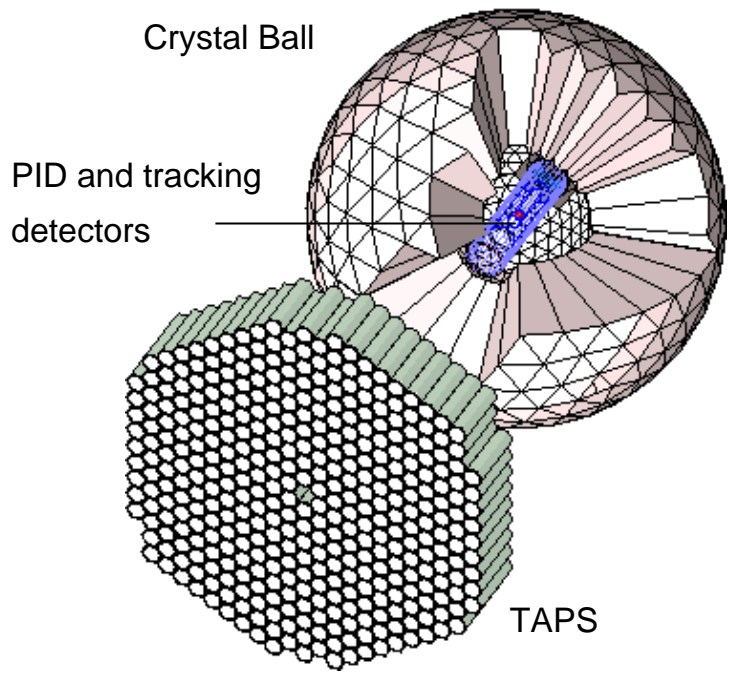

\subsection{Target Asymmetry}

The second measurement on the proton was to obtain the transverse target asymmetry, $T$. Data were taken in September 2010 and February 2011, and the analysis done predominantly by S. Schumann (Mainz-MIT), P. Hall Barrientos (Edinburgh), and P.B. Otte (Mainz). More details can be found in Ref. [2].

For a transversely polarized target and unpolarized beam, we have

$$
\frac{d \sigma}{d \Omega}(\theta, \varphi)=\sigma_{0}\left(1+P_{\mathrm{T}} T \sin \varphi\right)
$$

with the unpolarized differential cross section $\sigma_{0} \equiv d \sigma / d \Omega(\theta)$ and the target polarization $P_{T}$. The angle $\varphi$ denotes the orientation of the target spin relative to the reaction plane. The target asymmetry depends on the target polarization and is defined by

$$
T=\frac{1}{P_{\mathrm{T}} \sin \varphi} \cdot \frac{\sigma_{+}-\sigma_{-}}{\sigma_{+}+\sigma_{-}}
$$

where the $+/$ - denote target polarization parallel/antiparallel to the normal to the scattering plane. In principle, this can be measured as a counting-rate asymmetry

$$
T=\frac{1}{P_{\mathrm{T}} \sin \varphi} \cdot \frac{N_{+}-N_{-}}{N_{+}+N_{-}}
$$

because most of the quantities in the cross section cancel in the numerator and denominator, if properly normalized.

The set-up for this experiment was very similar to the one used for the beam-asymmetry measurement, except that a butanol frozen-spin target was employed to produce the polarized protons [6]. In addition, circularly polarized beam was used in order to obtain the beam-target asymmetry $F$, but that is not the topic of this work and will not be discussed. The specific run parameters are given in Table 3. 

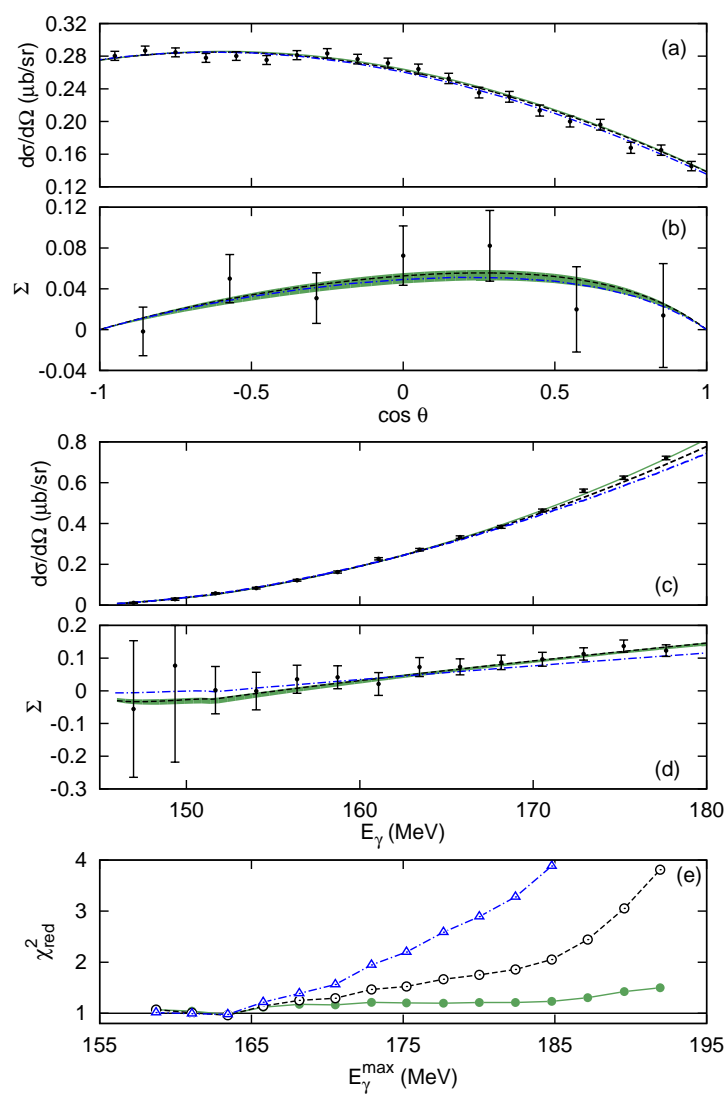

Figure 5: Differential cross sections in (a) $\mu \mathrm{b} / \mathrm{sr}$ and (b) photon asymmetries for $\pi^{0}$-production as a function of pion c.m. production angle $\theta$ for an incident photon energy of $163.4 \pm 1.2 \mathrm{MeV}$. Energy dependence of the (c) differential cross sections and (d) photon asymmetries at $\theta \simeq 90 \pm 3^{\circ}$. Errors shown are statistical only, without the systematic uncertainty of $4 \%$ for $d \sigma / d \Omega$ and $5 \%$ for $\Sigma$. The theory curves are black for HBChPT [4], blue for relativistic ChPT [5], and green for an empirical fit with an error band. (e) $\chi^{2}$ per degree of freedom for fits to the data in the range from $150 \mathrm{MeV}$ to $E_{\gamma}^{\max }$ for HBChPT [4] (open black circles), relativistic ChPT [5] (open blue triangles) and an empirical fit (solid green dots), with lines drawn through the points to guide the eye. Note that in (c) and (d), the two points in incident photon energy below the $\pi^{+}$threshold are included; these two points are excluded in the fits shown in (e) due to their large error bars.

The main experimental challenges come from the fact that the butanol target is made up of $\mathrm{C}_{4} \mathrm{H}_{9} \mathrm{OH}$, and so there are many sources of background. There is essentially one heavy nucleus for every two protons, and the detector is therefore swamped with $\pi^{0}$ s from $\mathrm{C}$ and $\mathrm{O}$, both coherent and incoherent. Even though the $\mathrm{C}$ and $\mathrm{O}$ nuclei are not polarized, they dilute the asymmetries:

$$
\begin{aligned}
A & =\frac{\sigma^{+}-\sigma^{-}}{\sigma^{+}+\sigma^{-}} \\
& =\frac{\left(\sigma_{p}^{+}+\sigma_{C}\right)-\left(\sigma_{p}^{-}-\sigma_{C}\right)}{\left(\sigma_{p}^{+}+\sigma_{C}\right)+\left(\sigma_{p}^{-}+\sigma_{C}\right)} \\
& =\frac{\sigma_{p}^{+}-\sigma_{p}^{-}}{\sigma_{p}^{+}+\sigma_{p}^{-}+2 \sigma_{C}} .
\end{aligned}
$$

Thus we need to know the lineshapes very well, and we must be able to eliminate effect of unpolarized, heavy nuclei. There were two main techniques for eliminating backgrounds: 1) the standard 

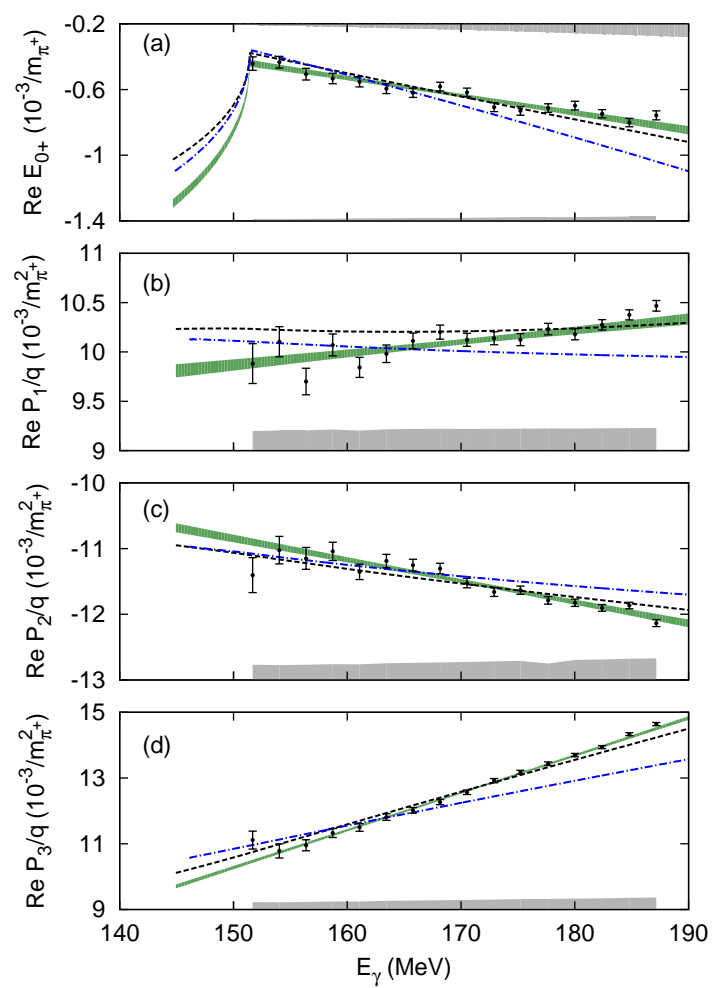

Figure 6: Empirical multipoles as a function of incident photon energy: (a) $\operatorname{Re} E_{0^{+}}^{\pi^{0} p}$, (b) $\operatorname{Re} P_{1} / q$, (c) $\operatorname{Re} P_{2} / q$, (d) $\mathrm{Re}_{3} / q$. The points are single-energy fits to the real parts of the $S$ - and $P$-wave multipoles, and the empirical fits from Eqs. (2.1) and (2.2) are shown with (green) statistical error bands. The \pm systematic uncertainty for the single-energy extraction is represented as the grey area above the energy axis, and the systematic uncertainty in the $S$-wave extraction due to the uncertainty in the size of the $D$-wave contributions is given by the grey area at the top of (a). The theory curves are the same as in Fig. 5.

technique of conducting a background subtraction by measuring the heavy-nucleus lineshape with a dedicated carbon-target run, and 2) calculating polarized cross sections and comparing them with theory. To do the former, one must properly normalize and subtract the heavy-nucleus contributions, and it is very tricky in the threshold region due to the huge coherent carbon cross section for neutral-pion photoproduction. The latter is the technique reported on here, and does not require carbon data at all, although one must know the normalization very well since nothing cancels as in the standard asymmetry calculations.

The product of unpolarized cross section and asymmetries is given by

$$
\sigma_{T} \equiv \sigma_{0} T=\frac{\sigma^{+}-\sigma^{-}}{P_{T} \sin \phi}=\frac{1}{P_{\text {eff }}^{y}} \frac{N_{\text {but }}^{+}-N_{\text {but }}^{-}}{\varepsilon \Phi_{\gamma} \rho_{p}} \frac{1}{2 \pi \sin \phi}
$$

and so there are no unpolarized contributions in the difference of $N^{+}$and $N^{-}$count rates:

$$
N_{\text {but }}^{+}-N_{\text {but }}^{-}=N_{p}^{+}+D X_{C}-N_{p}^{-}-N X_{C}=N_{p}^{+}-N_{p}^{-} .
$$

We can obtain polarized cross sections directly from butanol data, meaning there is no explicit background subtraction from carbon measurement. 
Table 3: Experimental parameters of the transverse-target measurement to obtain the imaginary part of the $S$-wave amplitude, $\operatorname{Im} E_{0^{+}}^{\pi^{0} p}$.

\begin{tabular}{l|c} 
PARAMETER & VALUE \\
\hline Electron Beam Energy & $450 \mathrm{MeV}$ \\
Target & Butanol \\
Radiator & Møller Foil \\
Tagged Energy Range & $100-400 \mathrm{MeV}$ \\
Channel Energy Resolution & $1.2 \mathrm{MeV}$ \\
Target Polarization & $\approx 80 \%$ \\
Beam on Target & $700 \mathrm{~h} \mathrm{C}_{4} \mathrm{H}_{9} \mathrm{OH}$ and $100 \mathrm{~h} \mathrm{C}$
\end{tabular}

In order to define the effective polarization, we define the following angle:

$$
\phi \equiv \phi_{\pi^{0}}-\phi_{T}
$$

where $\sin \phi>0$ defines + and $\sin \phi<0$ defines - . Thus

$$
P_{\text {eff }}^{y} \equiv P_{T}|\sin \phi|
$$

and we can place a cut $\phi$ to increase the effective polarization:

$$
|\sin \phi|>0.35
$$

which limits the angular coverage, but increases the polarization from about $50 \%$ to $60 \%$.

Neutral pions were identified detecting the two decay photons and forming an invariant mass, and then the incident photon energy was used to create missing mass distributions:

$$
m_{\mathrm{miss}}^{2}=(p+k-q)^{2}
$$

where $p, k$, and $q$ are the target, incident photon, and pion four-momenta, respectively. These missing mass distributions were calculated for each incident-photon energy bin, and for both polarization directions. Sample spectra can be seen in Figure 7.

To facilitate comparisons with theory, the following parametrization has been used:

$$
\sigma_{T}=\frac{q}{k} \sin \theta\left[t_{0} P_{0}(z)+t_{1} P_{1}(z)\right]
$$

where $P_{0}(z)$ and $P_{1}(z)$ are Legendre polynomials with $z=\cos \theta$, and coefficients $t_{0}$ and $t_{1}$. Sample polarized differential cross sections, $\sigma_{T}$, in four selected $1-\mathrm{MeV}$-wide energy bins are given in Figure 8. The Legendre coefficients are show in Figure 9.

The decomposition of $\sigma_{T}$, including the $D$-waves, is given by

$$
\begin{aligned}
\sigma_{T}=\frac{q}{k} \sin \theta\left\{3 \operatorname{Im}\left[E_{0^{+}}^{*}\left(E_{1+}-M_{1+}\right)\right]+\right. \\
3 \operatorname{Im}\left[4 E_{0^{+}}^{*}\left(E_{2+}-M_{2+}\right)-\right. \\
\left.\left.E_{0^{+}}^{*}\left(E_{2-}-M_{2-}\right)\right] \cos \theta\right\}
\end{aligned}
$$



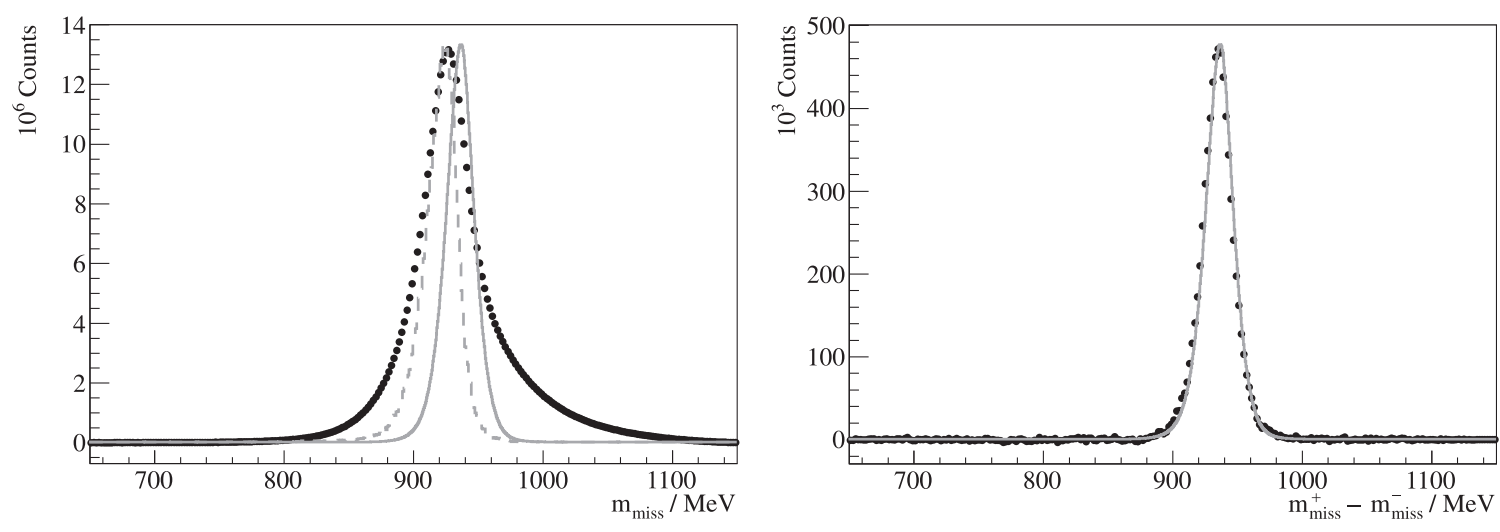

Figure 7: Left: Missing-mass distribution obtain from butanol target. Data points represent experimental results, while the grey solid and dashed lines are from MC simulations of $\gamma p \rightarrow p \pi^{0}$ and coherent $\pi^{0}$ production on ${ }^{12} \mathrm{C}$, respectively. Right: Polarization-dependent missing-mass distribution, where unpolarized contributions cancel and only counts from polarized protons remain. Data points represent experimental results, while the grey solid line is from an MC simulation of $\gamma p \rightarrow p \pi^{0}$.
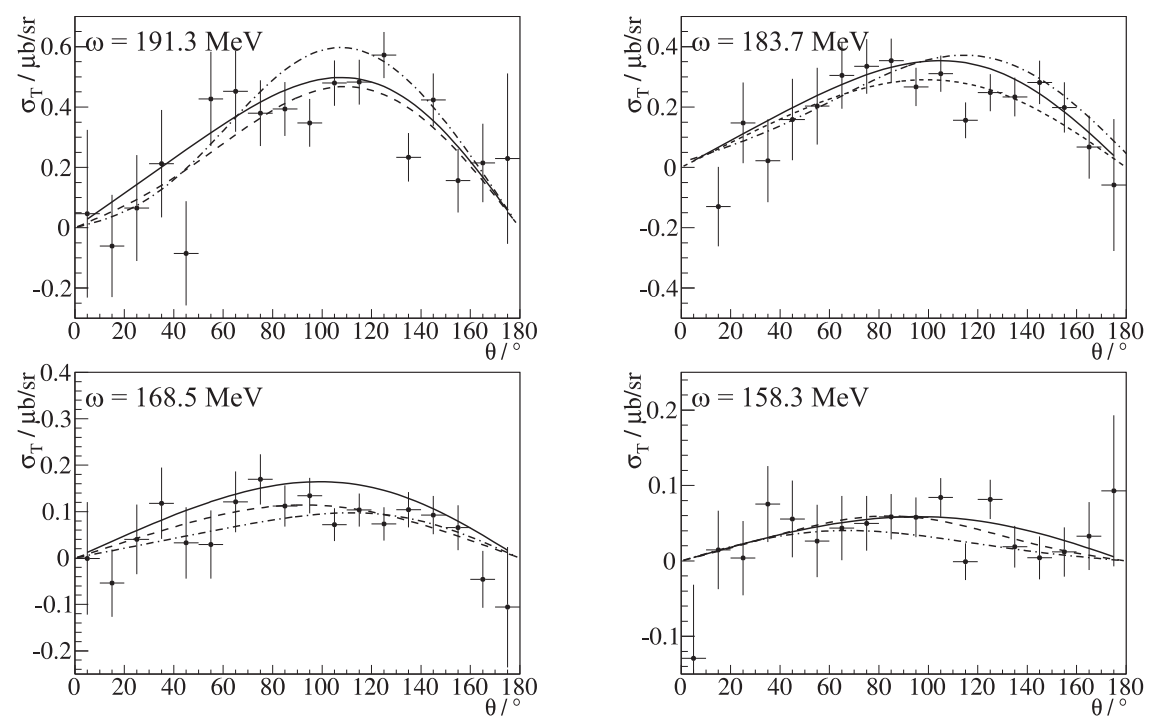

Figure 8: Polarized differential cross sections, $\sigma_{T}$, in four selected 1-MeV-wide energy bins are shown. The data points represent results with statistical uncertainty only. Solid lines are predictions of the DMT model [7], while the dashed and dashed-dotted lines show two-parameter Legendre fits to the experimental data and to the cross-check analysis [8], respectively.

where the real parts of the $S$ - and $P$-waves were taken from our previous experiment that measured $\Sigma$ and $d \sigma / d \Omega$. The imaginary parts of the $P$-waves were assumed to vanish in the threshold region, and the $D$-waves were included as fixed Born terms, meaning that $\operatorname{Im} E_{0^{+}}^{\pi^{0} p}$ was the only free parameter.

This is the very first experimental determination of $\operatorname{Im} E_{0^{+}}^{\pi^{0} p}$ and the results clearly show the rapid rise above the $n \pi^{+}$threshold as expected by the from the unitarity cusp. However, the data are below the naive parametrization with $\beta=3.35 \times 10^{-3} / m_{\pi^{+}}$indicating sensitivity to the energy 

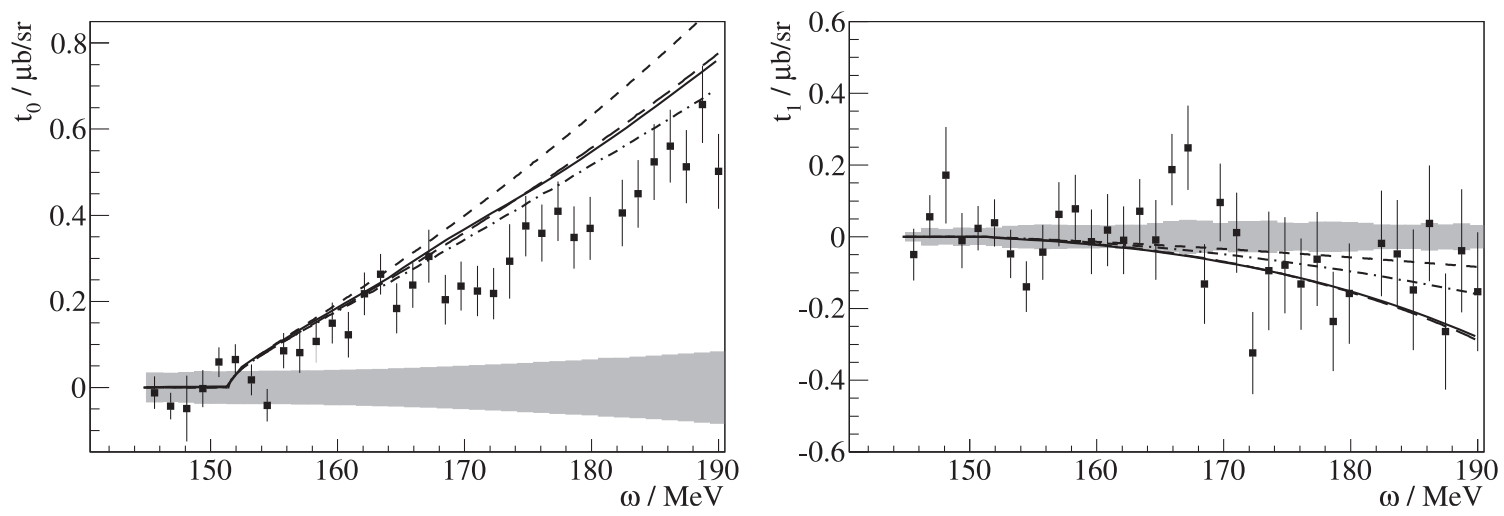

Figure 9: Legendre coefficients $t_{0}$ and $t_{1}$ for fits to $\sigma_{T}$. Error bars are statistical uncertainties and the grey bands show the absolute systematic uncertainties. The lines show the DMT model prediction [7] (solid), the multipole fit in Ref. [1] using the naive parametrization of $\operatorname{Im} E_{0^{+}}^{\pi^{0} p}=\beta(\omega) q_{\pi^{+}} / m_{\pi^{+}}$with a constant $\beta=3.35 \times 10^{-3} / m_{\pi^{+}}$(short-dashed), a prediction of Gasparyan and Lutz [9] (long-dashed), and the ChPT description in Ref. [5] (dashed-dotted).

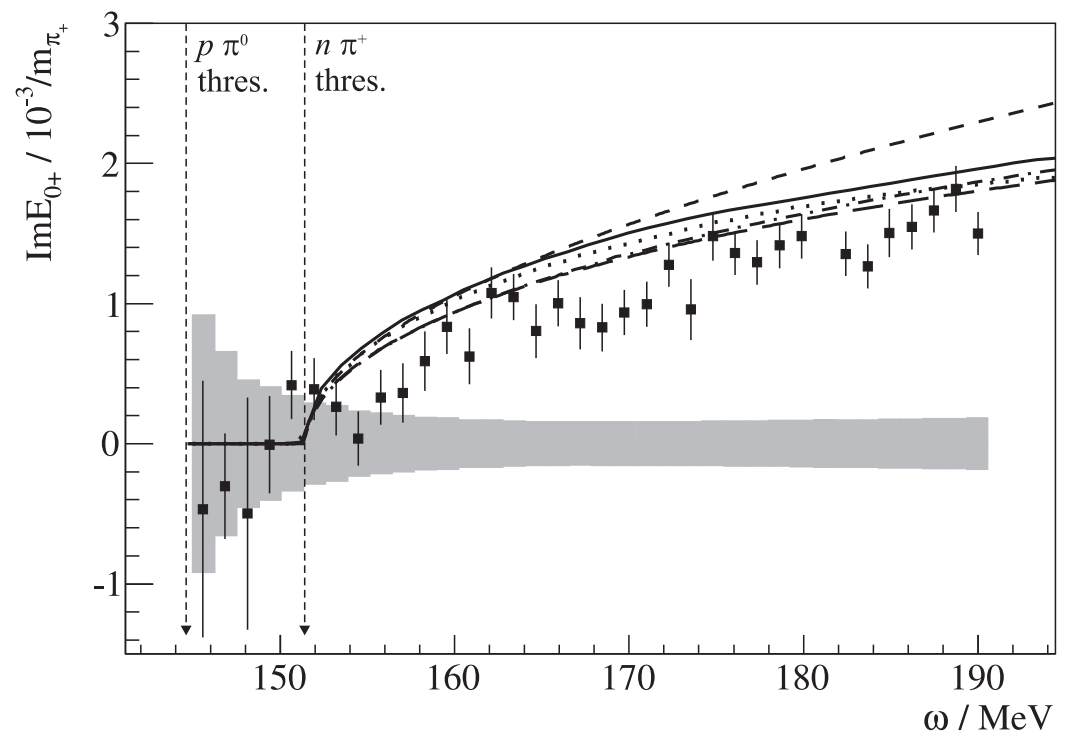

Figure 10: The imaginary part of the $E_{0^{+}}^{\pi^{0} p}$ amplitude from single-energy fits to the polarized cross section, $\sigma_{T}$. Data points are shown with statistical uncertainties (error bars) and absolute systematic uncertainties (grey shaded band). The lines are the DMT model prediction (solid), the naive parametrization with constant $\beta=3.35 \times 10^{-3} / m_{\pi^{+}}$(short dashed), a prediction. a prediction of Gasparyan and Lutz [9] (long-dashed), the ChPT description in Ref. [5] (dashed-dotted), and a HBChPT description from Ref [10] (dotted), scaled by a factor of 3.35/2.71 to match the unitary value of $\beta$ at the $n \pi^{+}$threshold. 
dependence of $\beta$.

Using the data and a two-parameter fit

$$
\beta(\omega)=\beta_{0}\left(1+\beta_{1} \cdot k_{\pi^{+}}\right) \quad \text { with } \quad k_{\pi^{+}}=\frac{\omega-\omega_{\mathrm{thr}}}{m_{\pi^{+}}}
$$

we obtain

$$
\begin{aligned}
& \beta_{0}=\left(2.2 \pm 0.2_{\text {stat }} \pm 0.6_{\text {syst }}\right) \cdot 10^{-3} / m_{\pi^{+}} \\
& \beta_{1}=\left(0.5 \pm 0.5_{\text {stat }} \pm 0.9_{\text {syst }}\right) .
\end{aligned}
$$

Unfortunately, large uncertainties preclude us from making a reliable determination of the energy dependence.

In conclusion, we stress again that this is the first measurements of $\sigma_{T}$ in neutral pion photoproduction in the threshold region, leading to a first direct measurement of $\operatorname{Im} E_{0^{+}}^{\pi^{0} p}$, confirming rapid rise above $n \pi^{+}$threshold. Uncertainties are still too large to determine a precise value of $\beta(\omega)$, but we plan on further running with transverse coil to improve statistics and therefore even smaller uncertainty in $\sigma_{T}$.

\section{Neutron}

As is usually the case, because there is no such thing as a free-neutron target, there is a dearth of information on the neutron photoproduction reactions; most information is obtained using nuclear targets. We have therefore proposed to measure the $\gamma+{ }^{3} \mathrm{He} \rightarrow{ }^{3} \mathrm{He}+\pi^{0}$ reaction near threshold in order to extract the $E_{0^{+}}^{\pi^{0} n}$ amplitude with the goal of testing confinement-scale QCD via Chiral Perturbation Theory. There is presently very little information on the S-wave $n \pi^{0}$ amplitude, and this experiment has the potential to change that. Recent theory work by Lenkewitz et al. [11] suggests that photoproduction of neutral pions from ${ }^{3} \mathrm{He}$ near threshold is highly sensitive to this quantity.

The S-wave amplitude $E_{0^{+}}^{\pi^{0} n}$ represents a crucial test of Chiral Perturbation Theory (ChPT), which predicts a faster rise of the total cross section for $\pi^{0}$ production on the neutron than for the proton, due to the fact that $\left|E_{0^{+}}^{\pi^{0} n}\right|>\left|E_{0^{+}}^{\pi^{0} p}\right|$. The convergence of the amplitude should be better for the neutron compared to the proton [12], making the prediction more reliable. Moreover, of the four photoproduction reactions on the nucleon,

$$
\begin{gathered}
\gamma p \rightarrow \pi^{0} p \\
\gamma p \rightarrow \pi^{+} n \\
\gamma n \rightarrow \pi^{0} n \\
\gamma n \rightarrow \pi^{-} p
\end{gathered}
$$

only reaction (4.3) has never been measured due to the difficulties associated with a free neutron target. The status of the S-wave amplitudes for photoproduction on the nucleon is given in Table 4 . Again, note the somewhat counter-intuitive ChPT prediction that $\left|E_{0^{+}}^{n \pi^{0}}\right|$ is roughly twice that of $\left|E_{0^{+}}^{p \pi^{0}}\right|$. 
Table 4: Results for the S-wave amplitude for the four photoproduction reactions on the nucleon (in units of $\left.10^{-3} / m_{\pi^{+}}\right)$.

\begin{tabular}{||c|c|c|c|c||}
\hline \hline Reaction & ChPT [12] & DR [13] & LET & Expt \\
\hline \hline$p \pi^{0}$ & -1.16 & -1.22 & -2.47 & $-1.33 \pm 0.08[3]$ \\
\hline$n \pi^{+}$ & $28.2 \pm 0.6$ & $28.0 \pm 0.2$ & 27.6 & $28.1 \pm 0.3[14]$ \\
\hline$n \pi^{0}$ & 2.13 & 1.19 & 0.69 & $? ? ?$ \\
\hline$p \pi^{-}$ & $-32.7 \pm 0.6$ & $-31.7 \pm 0.2$ & -31.7 & $-31.5 \pm 0.8[15]$ \\
\hline
\end{tabular}

The only way to overcome the absence of a free neutron target is to use a nuclear target and properly account for the both the proton and nuclear effects. Up to now, this has been attempted using coherent $\pi^{0}$ production from deuterium [17], but the nuclear effects were not disentangled and extraction of the free-neutron amplitude was not possible. Results for the deuteron S-wave amplitude, $E_{d}$, along with theory predictions are given in Table 5. One can see that final-state

Table 5: Results for the deuteron S-wave amplitude $E_{d}$ from coherent $\pi^{0}$ production from deuterium near threshold (in units of $10^{-3} / m_{\pi^{+}}$) [17].

\begin{tabular}{||c|c|c||}
\hline Method & $E_{d}$ & $E_{0^{+}}^{p \pi^{0}}+E_{0^{+}}^{n \pi^{0}}$ \\
\hline \hline LET & - & -1.78 \\
\hline ChPT $^{1}$ & $-1.8 \pm 0.2$ & 0.97 \\
\hline DR & - & -0.03 \\
\hline Expt [17] & $-1.45 \pm 0.04$ & - \\
\hline
\end{tabular}

interactions and meson-exchange currents are important, and therefore it is non-trivial to extract $E_{0^{+}}^{n \pi^{0}}$ using a deuteron target.

However, recent work by Lenkewitz et al. using the framework of Chiral Perturbation Theory to $\mathscr{O}\left(q^{4}\right)$ in two-nucleon corrections implies that a precise extraction of $E_{0^{+}}^{\pi^{0} n}$ using the coherent production of neutral pions on ${ }^{3} \mathrm{He}$ is possible [11]. This is shown in Figure 11 with the S-wave cross section for the reaction at threshold is given by:

$$
\left.a_{0} \equiv \frac{k}{q} \frac{d \sigma}{d \Omega}\right|_{q=0}=\left|E_{0^{+}}\right|^{2}
$$

where $k(q)$ is the c.m. momentum of the incident photon (outgoing pion), is plotted as a function of the S-wave amplitude for the neutron.

Note that there exist previous data of $\pi^{0}$ photoproduction on the ${ }^{3} \mathrm{He}$ reaction [18], but they were taken over 30 years ago without the benefit of a quasi-monochromatic photon beam and give total cross sections only (see Figure 12). As a result, they were unable to extract the neutron S-wave amplitude.

Before our experiment can run, however, the theory group needs to extend calculation to higher energies, above threshold. In addition we need to conduct proper rate calculations and carry out signal/background simulations with the high-pressure, active He gas target with special care taken 


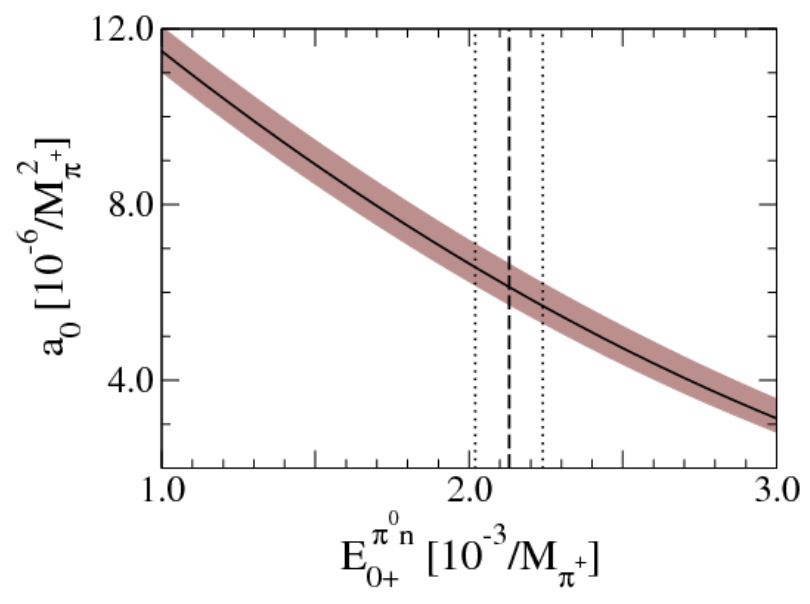

Figure 11: Sensitivity of $a_{0}$ for ${ }^{3} \mathrm{He}$ in units of $10^{-6} / m_{\pi^{+}}^{2}$ to the single-neutron multipole $E_{0^{+}}^{\pi^{0} n}$ in units of $10^{-3} / m_{\pi^{+}}$. The vertical dashed line gives the ChPT prediction for $E_{0^{+}}^{\pi^{0} n}$ and the vertical dotted lines indicate the $5 \%$ error in the prediction. The shaded band indicates the theory error estimated from the cutoff variation and a $5 \%$ error in $E_{0^{+}}^{\pi^{0} p}$ as described in Ref. [11].

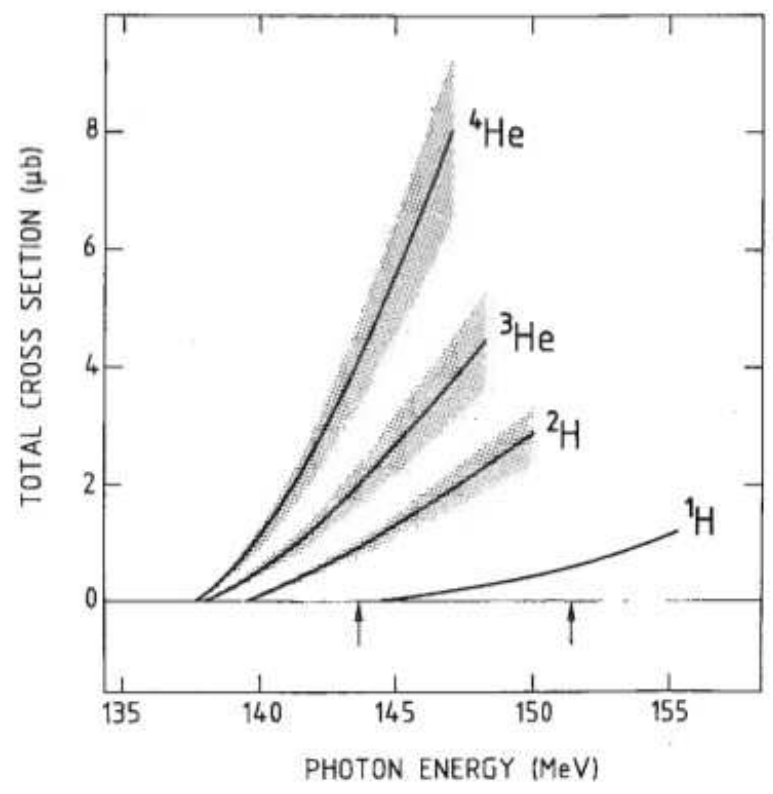

Figure 12: Previous data from total cross section measurements of near-threshold neutral-pion photoproduction on light nuclear targets [18]. 
Table 6: Experimental parameters of the proposed experiment to extract the neutron S-wave amplitude, $E_{0^{+}}^{\pi^{0} n}$, from near-threshold neutral-pion photoproduction on ${ }^{3} \mathrm{He}$.

\begin{tabular}{l|l} 
PARAMETER & VALUE \\
\hline Electron Beam Energy & $450 \mathrm{MeV}$ \\
Incident Photon ROI & $135-180 \mathrm{MeV}$ \\
Tagger Channel Energy Resolution & $1 \mathrm{MeV}$ \\
Radiator & $10-\mu \mathrm{m} \mathrm{Cu}$ \\
Collimator & $4 \mathrm{~mm}$ \\
Tagging Efficiency & $30 \%$ \\
\hline Target & Active, high-pressure ${ }^{3} \mathrm{He}$ gas \\
Thickness & $1.5 \times 10^{22}$ nuclei/cm ${ }^{2}$ \\
\hline Detector Configuration & $\mathrm{CB}+\mathrm{TAPS}+\mathrm{AT}$ \\
Detection Efficiency & $30-50 \%$
\end{tabular}

to examine the coherent vs. break-up channels. Expected parameters for the upcoming experiment are given in Table 6.

\section{References}

[1] D. Hornidge et al., Phys. Rev. Lett. 111, 062004 (2013).

[2] S. Schumann et al., Phys. Lett. B. 750, 252 (2015).

[3] A. Schmidt et al., Phys. Rev. Lett. 87, 232501 (2001); Phys. Rev. Lett. 110, 039993(E) (2013).

[4] C. Fernández-Ramírez and A. M. Bernstein, Phys. Lett. B 724, 253 (2013).

[5] M. Hilt, S. Scherer, and L. Tiator, Phys. Rev. C 87, 045204 (2013).

[6] A. Thomas, Eur. Phys. J. Spec. Top. 198, 171 (2011).

[7] S. S. Kamalov, S. N. Yang, D. Drechsel, and L. Tiator, Phys. Rev. C 64, 032201 (2001).

[8] P. B. Otte, Ph.D. thesis, Johannes Gutenberg-Universitä t Mainz (2015).

[9] A. Gasparyan and M. F. M. Lutz, Nucl. Phys. A 848, 126 (2010).

[10] V. Bernard, N. Kaiser, and U.-G. Meißner, Eur. Phys. J. 11, 209 (2001)

[11] M. Lenkewitz et al., PLB 700, 365 (2011) and EPJA 49, 20 (2013).

[12] V. Bernard, N. Kaiser, and U.-G. Meißner, Z. Phys. C 70, 483 (1996)

[13] O. Hanstein, D. Drechsel, and L. Tiator, Phys. Lett. B 399, 13 (1997)

[14] E. Korkmaz et al., Phys. Rev. Lett. 83, 3609 (1999)

[15] M. Kovash et al., $\pi \mathrm{N}$ Newsletter 12, 51 (1997)

[16] S.R. Beane et al., Nucl. Phys. A 618, 381 (1997)

[17] J.C. Bergstrom et al., Phys. Rev. C 57, 3203 (1998)

[18] P. Argan et al., Phys. Rev. C 21, 1416 (1980); Phys. Lett. B 206, 4 (1988). 